\title{
Chile, un caso de corrupción oculta
}

\author{
PATRICIO ORELLANA VARGAS ${ }^{\mathrm{a}}$
}

\section{Resumen}

Chile es considerado el país más probo (o menos corrupto) de América Latina y habitualmente, en los Índices de Percepción de la Corrupción de Transparencia Internacional en el Mundo figura en una excelente posición. Sin embargo, si se estudia el caso chileno con mayor detenimiento, se puede concluir que la corrupción está creciendo aceleradamente y la probidad que caracterizó al país ya es sólo un reflejo del pasado.

\begin{abstract}
Chile is considered the most honest (or less corrupt) country of Latin America and, it is normally ranked in an excellent position in the Indexes of Perception of Corruption of International Transparency in the World. However, if we carefully study the Chilean case, we can conclude that the level of corruption is dramatically growing and the probity that characterized the country is now only a remembrance of the past.
\end{abstract}

\section{UNA APROXIMACIÓN CONCEPTUAL}

La corrupción política y administrativa es un fenómeno social que ha existido permanentemente en las sociedades organizadas. En torno al poder político surgen, casi siempre, tendencias que implican que algunos que detentan parte de ese poder, lo usen para beneficiarse directamente, contraviniendo las normas impuestas y la legitimidad social. La definición escueta de $\mathrm{TI}^{\mathrm{b}}$ es la de Gianfranco Pasquino ${ }^{\mathrm{c}}$ que la define como el "fenómeno por medio del cual un funcionario público es impulsado a actuar de modo distinto a los estándares normativos del sistema para favorecer intereses particulares a cambio de una recompensa". Pero esta concepción no significa que este fenómeno ocurra siempre. Como en otros aspectos de la vida social es permanentemente la posibilidad, pero también existe como alcanzable de la probidad total, como lo indican los índices de percepción de la Corrupción de Transparencia Internacional que muestran países que alcanzan un puntaje de 10, que es el máximo (especialmente los países nórdicos). 
En este sentido la corrupción política y administrativa se refiere concretamente a lo que Transparencia Internacional y hasta la ley de Probidad y Transparencia definen como el desvío de recursos públicos que tienen finalidades sociales establecidas, pero que en virtud de maniobras ilegales, son traspasadas a particulares, que no son sus destinatarios legítimos. Son los fraudes, coimas, malversación de fondos públicos, triangulaciones, apropiación indebida, falsificaciones de documentos públicos, acceso a información privilegiada. etc.

\section{El proceso de corrupción}

En las últimas décadas en Chile ha habido una intensificación del proceso de corrupción política y administrativa y adquiere las más diversas formas, los casos más divulgados son los de Pinochet, que teniendo un poder absoluto traspasaba recursos públicos, como aquellos destinados a gastos públicos reservados, a sus cuentas bancarias personales. $\mathrm{O}$ el caso de generales que contrabandeaban armas a Croacia o Ecuador para obtener beneficios personales. Sin embargo, la corrupción pública y administrativa no es exclusiva de las dictaduras militares, los gobiernos democráticos la han seguido aplicando masivamente. Las investigaciones que se llevan a cabo sobre los desvíos de fondos del Ministerio de Obras Públicas, que formalmente aparecían como pagos de proyectos que debían ejecutar empresas privadas y que en la realidad nunca se realizaban y que implicaba que parte de esos recursos pagados eran destinados como sobresueldos a funcionarios de alto nivel del MOPT o a incrementar los fondos de campañas de determinados partidos o candidatos. Los caso son innumerables y en Chile los nombres de sobresueldos, indemnizaciones a todo evento, fondos contra el desempleo, capitación técnica, INDAP, CORFO, Correos, ESVAL, SENCE, Gendarmería, Aduanas, venta de empresas públicas, honorarios, horas extraordinarias, proyectos, etc. configuran el vocabulario común que tiene contenidos directos con el fenómeno de la corrupción.

A pesar de que la corrupción es muy dificil de perseguir y procesar judicialmente, entre 1983 y el año 2003 había 282 procesos en los tribunales ${ }^{\text {d }}$ sobre estos casos, que afectaban a entidades de todos los niveles del sector público. Entre los procesados y condenados había ministros, subsecretarios, diputados y presidentes de partidos. Esto no había ocurrido jamás en la historia de Chile y también puede ilustrarse con la información sobre el número de artículos sobre corrupción aparecidos en la prensa, mientras que en el año 2001 se publicaron 1.717 artículos, en el año 2006 aparecieron 4.198. e $^{\circ}$

Sin embargo, la corrupción no ha sido una característica esencial de la historia de Chile. Por el contrario, si se revisan las obras de los principales historiadores, este fenómeno es excepcional y más aún existe el convencimiento de que la probidad pública fue una característica de la historia republicana de Chile. Esto no significa una tesis de probidad absoluta, pues hay aspectos que nunca lo fueron, como los sistemas electorales y los procedimientos en los nombramientos de funcionarios públicos y la relación entre negocios y política, que siempre pueden objetarse $\mathrm{f}^{\mathrm{f}}$. 


\section{Dictadura y corrupción}

En otros escritos hemos sostenido que esta historia de relativa probidad-ejemplar si se considera comparativamente con el resto de América Latina- tiene un punto de quiebre. La incorporación de la corrupción masiva, practicada por muchos de los políticos y jefes administrativos, ocurre cuando el sistema de control y equilibrios, propios de la separación de poderes, desaparece y es reemplazado por la concentración de todos los poderes en el Ejecutivo, lo que ocurre durante la dictadura militar. Se cumpla la premisa que Lord Acton estableció como principio en la ética pública: el poder dictatorial lleva a la corrupción total. En sus palabras: "el poder absoluto corrompe absolutamente".

La ausencia de una justicia independiente, la inexistencia de la fiscalización parlamentaria y el funcionamiento timorato de la Contraloría, permitió una corrupción sin límites, cuyos casos más conocidos son los del enriquecimiento de la familia de Pinochet, la generación de grandes fortunas por al traspaso a manos privadas de las empresas públicas, el contrabando de armas practicado por militares y la implantación de los sobresueldos e indemnizaciones a todo evento para los políticos que ocuparon altos cargos. Los otros factores sociales que facilitaron este proceso corrompente es la ausencia de prensa libre, sólo Fortín Mapocho, ANÁLISIS y APSI, al final de la dictadura, fueron capaces de develar algunas de las facetas de la creciente corrupción. Finalmente la sociedad civil había sido totalmente desarticulada y no tenía ninguna capacidad orgánica de reaccionar.

Siempre se ha destacado que en la historia de Chile, ningún primer mandatario se enriqueció cuando estaba en el poder y son legendarias las figuras de O’Higgins, Carrera, Ramón Freire, Manuel Montt, Aníbal Pinto, y muchos de los presidentes del siglo XX por su probidad pública como Pedro Aguirre Cerda, Jorge Alessandri, Eduardo Frei Montalva o Salvador Allende. El primer caso de quien se enriqueció en el poder en términos extraordinarios es el de Pinochet.

Algunos críticos de esta visión consideran que es una forma de justificar la corrupción durante la restauración de la democracia. No se trata de justificar la corrupción ni en dictadura ni en democracia, pero interesa ver cómo se desarrolló en Chile, precisamente porque sólo conociendo el proceso es posible combatirla eficientemente.

\section{La ideología de la corrupción}

La caracterización e identificación de los casos de corrupción es una tarea de toda la sociedad. No puede limitarse a las definiciones legales que sirven para procesar y en algunos casos, sancionar a los corruptos, pues estas situaciones son la excepción. En la realidad chilena se ha desarrollado una cultura de la corrupción, una de cuyas manifestaciones fue la llamada "ideología de la corrupción" que surgió cuando en el 2007, destacados líderes políticos (Schaulson del PPD, Martner del PS, y Boeninger 
del PDC) reconocieron que los partidos políticos de gobierno financiaban sus campañas con fondos fiscales, que a través de diversos procedimientos desviaban de sus objetivos sociales, para traspasarlos a las arcas partidarias o a sus bolsillos personales. Los casos más conocidos son los falsos proyectos (especialmente estudios) que son pagados como si se hubiesen realizado o triangulaciones consistentes en pagos a empresas privadas que en parte devuelven a los jerarcas de los ministerios o servicios para que se pagaran los sobresueldos o financiaran las mencionadas campañas.

\section{DEMOCRACIA Y CORRUPCIÓN}

La democracia que recomenzó con el gobierno de Patricio Aylwin introdujo algunos cambios sustanciales en el funcionamiento del sistema político. Sin embargo, gran parte de las instituciones establecidas durante la dictadura se mantuvieron por la debilidad de los acuerdos para restablecer la democracia y por factores de carácter ideológico que fueron esenciales. Entre ellos se destacan: la debilidad inicial, el relativismo ético la continuidad burocrática, y esencialmente la ausencia de un proyecto político integrado.

\section{La debilidad inicial}

En primer lugar, el golpe militar significó una derrota estratégica de la izquierda en Chile, lo que se acrecentó con el derrumbe del socialismo en el mundo. En estas condiciones la izquierda carecía de poder para intentar lograr un apoyo mayoritario. Por otra parte, el centro político, representado principalmente por la Democracia Cristiana, había tenido un comportamiento voluble, apoyando inicialmente el golpe militar, pero recuperando sus valores democráticos posteriormente, tampoco estaba legitimada popularmente y su concepción de una sociedad comunitaria se había desvanecido. En estas condiciones la derrota de la dictadura fue parcial y ambos bloques, izquierda y centro, consideraban que lo que importaba era reconquistar la democracia, aunque muchas de las condiciones impuestas por la dictadura significaban aceptar una democracia muy limitada, donde sólo existirían dos bloques políticos: la derecha que tenía un respaldo próximo al 40 \% de la votación y la Concertación de Partidos por la Democracia que a duras penas superaba el 50\%.

El gobierno de Aylwin fue el resultado de un plebiscito, en el cual si bien la dictadura fue rechazada, mostró un apoyo popular que superaba ampliamente al tercio que tradicionalmente la derecha había obtenido en los comicios. Esta situación y el poder militar controlado por Pinochet determinaron una debilidad intrínseca en el primer gobierno de la Concertación.

\section{El relativismo ético}

Desde el punto de vista de nuestro análisis conviene resaltar la posición ética de la Concertación. La manipulación del poder político obtenido, rápidamente mostró sus 
limitaciones, ya que Pinochet y la derecha conservaba una cuota esencial del poder, especialmente el militar y económico. La solución teórica fue olvidar la ética de los principios que implicaba cambios trascendentales, especialmente en el plano de los derechos humanos, la distribución del ingreso y el rol del Estado. La solución fue la adhesión al relativismo ético, expresado en la ética de la responsabilidad de Max Weber $^{s}$. Ética pragmática que se fundaba en la real politik, que en nuestro concepto había sido la que pavimentó el ascenso de Hitler al poder durante la república de Weimar. El divulgador de esta posición fue un alto funcionario socialista ${ }^{\mathrm{h}}$.

Esta posición ética, la sintetizo el presidente Patricio Aylwin cuando presentó el Informe Rettig a la población, acuñando la frase que lo identificó éticamente. "justicia en la medida de lo posible". Lo que implicó divulgación de algunos excesos de la dictadura igualándolos conceptualmente a las acciones armadas realizadas por los que intentaron defender el gobierno constitucional, además de pagar algunas indemnizaciones mínimas a los familiares de las víctimas de la represión.

Más tarde, el presidente Frei Ruiz Tagle prosiguió aplicando la misma concepción ética y la profundizó en el campo de los derechos humanos y especialmente en la lucha contra la corrupción. Su ley de Probidad y Transparencia fue aprobada por unanimidad. Lo que implicó que las posiciones más diversas y contradictorias se incluyeran en esa ley, como por ejemplo la prohibición de los funcionarios públicos de recibir regalos y más adelante la autorización de recibirlos, si lo aconsejaba la costumbre, la cortesía, la buena educación, el protocolo y otros factores muy imprecisos. También se establecía la obligación de establecer una política de transparencia a los servicios públicos y al mismo tiempo autorizaba por decisión administrativa establecer numerosas excepciones. Finalmente, si un servicio no otorgaba la información solicitada se podía recurrir a los tribunales, con los costos y demoras que ello implicaba, pero los jefes de servicios podían, de todas maneras, seguir negando la información, si que hubiese ninguna sanción.

Finalmente, el presidente Lagos declaró que tendría una política muy decidida en contra de la corrupción y la caracterizó con la frase de sancionar "caiga quien caiga". Pero en realidad, para enfrentar la crisis moral que significaba el descubrimiento de la corrupción generalizada en sobresueldos, honorarios, indemnizaciones a todo evento, etc., llegó a un acuerdo con el líder de la derecha, señor Longueira y se resolvió el problema dictando una ley que legalizó los actos de corrupción mencionados.

\section{La continuidad burocrática}

Este fue un factor que dificilmente se ha mencionado. Reviste dos aspectos, uno relativo a la naturaleza conservadora de la burocracia y el otro coyuntural que corresponde a las justificaciones morales elaboradas.

La burocracia tiene como una de sus características esenciales el ser un sistema de dominación basado en la jerarquía. El burócrata, esencialmente, en el caso chileno, 
es el político que ha sido nombrado en un cargo de jefatura y ejerce el poder desde ese cargo en cualquier nivel. Como estamento social definido, legitima su acción en virtud de que ha sido nombrado por una autoridad superior elegida democráticamente, aunque intenta crear otra legitimidad basada en sus estudios (si los tiene) y en su capacidad personal. En estas circunstancias el burócrata (jefe) intenta por todos los medios recibir una remuneración acorde a su posición y privilegios. Masivamente estos burócratas encontraron en la doctrina administrativa norteamericana la teoría del gerente público, que exalta el rol de los jefes y le asigna el rol estelar de agente de los cambios, lo que difícilmente calzaba con la realidad chilena, pero que fue asumida por los gobiernos de la Concertación. Uno de su ministros la caricaturizó con la frase "si uno paga con maní... sólo puede contratar monos" (lo que era una copia de la... declaración de un ministro de Singapur). De esta manera justificaban moral y técnicamente el recibir el doble de las remuneraciones legales. Para ello crearon un complejo sistema de triangulaciones que se describe más adelante.

El otro factor esencial fue que la dictadura mantuvo en sus cargos a sus burócratas porque dictó una ley de inamovilidad para ellosi. Los nuevos jefes "democráticos" debieron compartir con ellos y se logró la necesaria continuidad de la cultura burocrática. Uno de los elementos esenciales de esta cultura era que durante la dictadura los sobresueldos y las indemnizaciones a todo evento eran instituciones establecidas sin ningún obstáculo ético. Rápidamente los nuevos jefes consideraron una fortaleza a su favor mantener estos sistemas de privilegio y lo lograron con gran facilidad, de manera que la corrupción de la dictadura fue recibida con el entusiasta beneplácito de los nuevos jefes burócratas.

Finalmente, el otro factor esencial que explica la corrupción generalizada, que siendo heredada de la dictadura se continuo en la democracia, es la ausencia de un proyecto político general. En efecto, el Frente Popular tenía un proyecto de industrialización y desarrollo del país, la Democracia Cristiana, durante el gobierno de Frei Montalva, tenía un proyecto comunitario que significaba reforma agraria, sindicalización, organización de los pobres, etc. Allende tenía un proyecto de reformas estructurales y hasta Jorge Alessandri tenía un proyecto de liberalismo económico. Pero la Concertación de Partidos por la Democracia carecía efectivamente de un proyecto, excepto la recuperación de la democracia, pero desde el primer momento aceptaron el modelo neoliberal como el único viable. La paradoja resultante fue que la derecha que había perdido las elecciones tenía un gobierno de otro signo que, sin embargo, aplicaba con mucho entusiasmo su propio modelo. Parece que perdiendo habían ganado.

Este fenómeno, explicado estructuralmente y coyunturalmente por algunos de los factores analizados, determinó que en Chile estemos en presencia de lo que Weber ${ }^{j}$ caracterizó como gobierno de "el poder por el poder", que existe sólo para aprovechar el poder, pero no para llevar a cabo algún proyecto trascendental. 


\section{CARACTERÍSTICAS DE LA CORRUPCIÓN. ${ }^{k}$}

Hay varios factores que permiten que numerosos casos de corrupción queden cubiertos con un manto de opacidad que deja a los corruptos en la impunidad.Aunque advertimos que esta lista no es taxativa:Algunos de estos factores o mecanismos son:

- La complejidad de las tramas,

- El transcurso del tiempo,

- El formalismo legal,

- La salida temporal de la escena,

- La irresponsabilidad jerárquica,

- La eliminación de testigos,

- Las maniobras de reestructuración,

- La falsificación de pruebas,

- La jurisprudencia voluble,

- La opacidad burocrática,

- La levedad de las sanciones,

- La premiación de los involucrados,

- La justificación moral,

- El acuerdo político, y,

- La legalización de la corrupción.

Estas formas de eludir la responsabilidad en los delitos de corrupción, generalmente se presentan combinadas en intrincadas relaciones y son utilizadas por la clase política en su conjunto, tanto por políticos de gobierno como de oposición, así como por la alta burocracia civil y militar; por los directivos de la administración central y también por los de las municipalidades y empresas estatales. Estos procedimientos se han utilizado durante la dictadura militar de la derecha así como durante el gobierno democrático.

Cada uno de estos mecanismos merece un análisis detallado, pero han sido explicitados en centenares de casos investigados por jueces y por los periodistas, forman parte del léxico de la corrupción y han sido estudiados por los investigadores preocupados de la ética pública. 
Revista de Sociolocía 21 / 2007 Chile Hoy

Facultad de Ciencias Sociales - Universidad de Chile

\section{La complejidad de las tramas}

Los casos de corrupción, para operar exitosamente, deben ser diseñados tomando toda clase de precauciones y con un conocimiento cabal de los marcos legales e institucionales. Un ejemplo claro es la investigación del juez Cerda sobre las cuentas del Banco Riggs y el fraude al fisco de Pinochet y sus cómplices. La investigación actualmente cuenta con decenas de miles de hojas y sólo fue posible a partir de la investigación de una gran capacidad técnica (el FBI), así como la investigación del Senado norteamericano sobre cuentas bancarias de organizaciones terroristas.

Esta complejidad es una selva intrincada que permite a los corruptos escabullirse de las responsabilidades penales y civiles de los delitos.

\section{El transcurso del tiempo}

Derivado en gran medida de la complejidad de estos actos delictuales, otro factor es el tiempo que se consume en las investigaciones, el que tiene efectos de diverso tipo. Ejemplos de ello son la investigación que lleva a cabo la ministra Gloria Ana Chevesich sobre el MOPT que ya lleva 5 años de diligencias o el proceso por pagos excesivos de desmalezamiento de la ENAP que cumplió 14 años y que aún quedan recursos procesales pendientes.

Estas demoras son factores que aminoran la gravedad de los hechos, pues la preocupación de la opinión pública no puede mantenerse tanto tiempo y los hechos y procesos quedan en la tenue sombra del olvido. Pero el efecto más importante y operativo es la prescripción, que es la gran puerta por donde se escabullen los corruptos. En efecto si el proceso no se inició en el momento oportuno, por el mero hecho del transcurso del tiempo, el delito queda sin efecto y no hay sanción. Un caso ilustrativo es el de Guillermo Díaz, subsecretario de Transportes, que fue recibido con aplausos en una asamblea del Partido Demócrata Cristiano, no por ser absuelto del cargo de corrupción, sino porque su proceso había quedado prescrito por el mero transcurso del tiempo, (sin embargo, la decisión de la Corte de Apelaciones de Santiago cambió y el proceso ahora sigue). Pero hay otros casos en los que el delincuente ha quedado libre por la puerta ancha y cómoda de la prescripción que, además, ni siquiera debe solicitarse ya que debe aplicarse obligatoriamente y rige en forma automática.

\section{El formalismo legal}

La esencia de la impunidad en los casos de corrupción es la tradición del sistema judicial chileno, que sólo concibe el delito si éste está clara y precisamente definido en la ley. Por lo tanto, se dejan de lado los principios generales del derecho, la doctrina y la ética no tiene la menor cabida en esta maquinaria. Es una justicia sin ética.

El caso más destacado es la reciente aceptación del recurso de amparo de algunos familiares y secuaces de Pinochet porque el fraude al fisco sólo puede ser cometido 
por funcionarios públicos, si lo hace un particular, ese delito no se configura. Otro ejemplo es la condena de Ricardo Farías, asesor del senador Girardi, por presentar facturas falsas como gasto electoral, sin que ello afecte al senador Girardi, aunque eran declaraciones de su comando electoral.

\section{La salida temporal de la escena}

En el ámbito de la realidad política y especialmente en el escándalo que genera el acto de corrupción ante la opinión moral de la sociedad, conviene salir de la escena pública y en muchos casos, hasta implica un premio. Los operadores políticos saben que se puede lavar la imagen sacando temporalmente al afectado de la escena, especialmente si no ha habido proceso o si se ha eludido la sanción. Caso ilustrativos es el de INVERLINK, sociedad que estafó a la CORFO en millones de dólares, cuyo presidente, Alvaro García, ex ministro (quien dice no saber lo que hacia su empresa) fue enviado como embajador a Suecia por un tiempo y ahora regresó con su nueva imagen. El ex presidente del PPD,Víctor Manuel Rebolledo, culpable en el caso coimas, sigue en su Partido, el que no lo sancionó y sigue en la política activa en su provincia, esperando a que pase el temporal moral. El ex ministro de Bienes Nacionales, Jorge Heine que debió renunciar a su cargo por utilizar indebidamente recursos fiscales, fue designado embajador en la India. El mencionado Guillermo Díaz fue trasladado al cargo de presidente de EFE para desvincularlo de sus otras actuaciones en el MOPT.

\section{La irresponsabilidad jerárquica}

Otro de los mecanismos más utilizados es eludir la responsabilidad de los actos de corrupción, traspasándola a los subalternos. La excusa habitual es que no se tenía conocimiento de los actos que realizaban sus dependientes. Esto ocurre en varios casos de los fondos fiscales para los programas de empleo que fueron utilizados en campañas electorales. Esta es la situación aparentemente de los diputados del PPD, Rodrigo González y Laura Soto, pero que también es empleada en muchos otros casos como el de Girardi del PPD y Sebastián Piñera Lily Pérez, de Renovación Nacional, que presentaron facturas falsas en sus rendiciones de cuentas al Registro Electoral. Una variante de esta actitud, es justificar algunos hechos porque fueron decisiones de los asesores respectivos, en circunstancias de que la esencia de la asesoría consiste en la recomendación de una o varias opciones que se sugiere al ejecutivo, pero quien decide en definitiva es quien tiene la calidad de ejecutivo y por lo tanto asume la responsabilidad de su elección.

\section{La eliminación de testigos}

La forma más criminal de eludir la responsabilidad por actos de corrupción es sin duda, la eliminación de testigos o de cómplices arrepentidos. Esta situación es la del coronel Huber, quien sabía sobre el contrabando de armas a Croacia que afecta a varios generales y oficiales del Ejército. Para silenciarlo se le asesinó. Es posible que este 
procedimiento haya sido utilizado en el caso de la triangulación de fondos del MOPT y la Universidad de Chile a través de IDECOM y CIADE. En este caso, uno de los implicados, Enrique Ponce León, aparece como suicidado y de otro, Daniel Figueroa, se encontró su cadáver en Pirque. Hay otros casos similares en investigación.

\section{Las maniobras de reestructuración}

Otro de los procedimientos más impúdicos es el de realizar amplios programas de re-estructuración de los servicios acusados de corrupción, para aparentar de que se está haciendo una limpieza total, aplicando leyes vigentes desde la época de la dictadura. Es el caso de la Facultad de Economía de la Universidad de Chile, donde se exoneró a dos asistentes sociales, una secretaria de dirección de departamento grado $9^{\circ}$, un profesional de la planta directiva grado $5^{\circ}$ y un encargado de cobranzas, todos ellos funcionarios con más de 24 años de servicio, con cargos en propiedad y además, el encargado de encuestas. Ninguno de ellos tenía acceso al plano de las decisiones que se adoptaron en la triangulación de fondos (más de mil millones de pesos, unos dos millones de dólares), que fue un acuerdo entre ministros, rector, decanos, jefes de servicio y directores de departamento y director de IDECON. Estos despidos se hicieron sin realizar ninguna investigación ni sumario administrativo, simplemente aplicando la Ley Federici de la dictadura (nombre del rector militar de la Universidad durante la dictadura). Estas exoneraciones, en algunos casos, ni siquiera consideraron la indemnización legal, la que ha sido exigida hasta por la Contraloría General de la República, obligación que hasta ahora se ha eludido.

\section{La falsificación o eliminación de pruebas}

En estos procesos es habitual presentar documentos falsos, como es el caso de las facturas mencionadas o el de Matías de la Fuente y Sergio Cortés condenados por falsificar instrumento público en el caso MOPT. En este ministerio habían desaparecido hasta computadores con información.

\section{La jurisprudencia voluble}

En Chile, ni la jurisprudencia ni la doctrina obligan a los jueces, de manera que un juez puede resolver un caso similar a otro de manera totalmente distinta a la sentencia del primero. Esto genera confusión y facilita las presiones que puedan existir y los fallos pueden ser contradictorios. En los países anglosajones la jurisprudencia es obligatoria y se consideraría un absurdo judicial que un mismo hecho recibiera sentencias diametralmente distintas. En Chile eso ocurre permanentemente, como, por ejemplo en la aplicación o no de la Amnistía, en los casos de Derechos Humanos y hasta en la aceptación o rechazo del Derecho Internacional

\section{La opacidad burocrática}

La Administración Pública chilena se ha caracterizado, durante décadas, por su culto del secretismo. La burocracia se fundamenta en la jerarquía y para que ella subsista 
con sus privilegios y potestad para operar, requiere hacer inoperante la transparencia. A pesar de la legislación vigente y los nuevos proyectos a punto de aprobarse, todos los altos niveles burocráticos se niegan a entregar información significativa y sólo traspasan información propia de sistemas de relaciones públicas, consistente en datos inocuos o vagos. Hay normas aún vigentes que obligan a los funcionarios a guardar secreto.

A pesar de que existen sentencias judiciales al respecto, los jefes de servicio no entregan información, como ocurre, por ejemplo, con el Servicio de Aduanas, el que ha sido obligado por sentencia judicial a entregar información solicitada por un empresario sobre la importación de mascarillas anti tóxicas y Aduana sigue sin entregarla. La Comisión Investigadora del Transantiago ha presentado listas de servicios, en especial del MOPT que no ha remitido la información solicitada y los parlamentarios de oposición han presentado listas de 45 casos en que no se entrega la información solicitada. Todo esto, a pesar de existir una política pública de transparencia y de instrucciones específicas de la Presidenta para facilitar la información a los servicios públicos.

La falta de transparencia es el mejor manto para ocultar la corrupción.

\section{La levedad de las sanciones}

Otro factor que incide en los niveles de corrupción es la levedad de las sanciones, tanto sociales como penales y administrativas.

En varios de los casos de la generalizada corrupción en el MOPT, las penas impuestas son muy reducidas, generalmente algunos días de penas remitidas, esto es, la firma periódica en algún recinto, gozando de casi plena libertad. Estas penas se establecen en virtud del mérito de "intachable conducta anterior" otra variante es la libertad vigilada, así por ejemplo el ex ministro de obras públicas Carlos Cruz, militante del Partido Socialista. Fue condenado a 4 años de libertad vigilada, pero la corte superior se la rebajó a 61 días.

En otros casos, las normas establecen que en los sumarios administrativos, la Contraloría puede proponer sanciones, pero su ejecución queda a la discrecionalidad del jefe administrativo superior, así por ejemplo Germán Molina, ex ministro, que había firmado asistencia a reuniones pagadas que no se realizaron, fue sancionado por la Contraloría, pero el Ministro respectivo (de su mismo partido) dejó pasar los plazos y no hubo sanción y Molina fue promovido el Consejo de Administración de EFE y de otras empresas. Además es asesor en ética de la directiva del PPD.

\section{La premiación de los involucrados}

Parece increíble reconocer que muchos de los personeros involucrados de una u otra forma en actos de corrupción son premiados por sus partidos o por las autoridades 
políticas, así ocurre con algunos que ya se mencionó, al ser nombrados embajadores o presidentes de empresas del Estado. Otros, son nombrados en comisiones especiales de probidad como los casos de García y Molina que son asesores en probidad de la directiva del PPD y Jorge Heine que era presidente del tribunal ético de ese mismo partido. El senador Girardi, que se ha visto envuelto en varios casos de corrupción, fue reincorporado a la directiva del PPD y hasta fue en misión oficial a saludar al Papa a Roma. Olivia Recal, propietaria de la empresa involucrada en la triangulación de fondos IDECON-CIADE-MOPT, fue nombrada Subsecretaria de Hacienda y dirige una comisión de Probidad.

\section{La justificación moral}

La corrupción es una ideología tan fuerte que hasta pretende encontrar justificaciones morales, las que si bien no se expresan públicamente, existen a sotto voce. Así es evidente que los partidos de gobierno justifican la corrupción como el único medio de obtener fondos para sus campañas electorales, mientras que los partidos de derecha, tienen aseguradas una inmensa cuantía de recursos por parte de los empresarios. De esta manera se llega a sostener la paradoja de que la corrupción es la única garantía para mantener la actual democracia.

Otra justificación muy empleada fue la teoría del gerente público, en virtud de la cual se sostenía que los directivos públicos debían ser muy bien pagados para que fueran eficientes.

Al mismo tiempo, los políticos de gobierno justifican la corrupción argumentando con hechos, que durante la dictadura, cuando había un poder absoluto, la corrupción era también absoluta, como lo demuestra el caso de Pinochet y sus cúpulas políticas y empresariales que se enriquecieron indebidamente. Se trataba del primer caso de un mandatario corrupto en toda la historia de Chile.

A su vez, los de oposición, argumentando con hechos, muestran la corrupción actual y la atribuyen al sistema partidario y la ausencia de valores.

Estas falsas explicaciones no justifican ni lo uno ni lo otro. El empate en casos de corrupción no libera ni a los políticos de gobierno concertacionista ni a los partidarios de la dictadura, que constituyen la actual oposición de derecha, de sus respectivas responsabilidades. La corrupción no tiene justificación en ningún caso.

\section{El acuerdo político}

A pesar de las apariencias, la violencia de la derecha para combatir la corrupción es mucho menor a la aparente. En realidad no cumple el rol fiscalizador que le corresponde a una oposición. Lo que parece inexplicable, pero tiene sus fundamentos en que los sobresueldos, las indemnizaciones a todo evento y otras formas de corrupción, son engendros creados durante la dictadura de la derecha y que siguieron aplicándose durante la democracia.Además la derecha está interesada en preservar la imagen de un 
Chile honesto, imprescindible para los negocios internacionales que son su campo de acción predilecto, por lo tanto, está también interesada en ocultar la corrupción ante los ojos del mundo. Esta coincidencia entre gobierno y oposición es lo que permite que la calificación de Chile como país probo en los índices de Transparencia Internacional sea tan positiva porque tanto gobierno como oposición ocultan los casos de corrupción.

\section{La legalización de la corrupción}

La prueba más fehaciente de la coincidencia oposición-gobierno en el tema de la corrupción fue el acuerdo Lagos-Longueira, que legalizó los actos corruptos. Según la ley, los sobresueldos (principal expresión de la corrupción) pasaron a ser legales. Es decir, una costumbre corrupta, fue purificada por la santificación legal aprobada por todo el sistema político, incluyendo gobierno y oposición. Además, el Director de Impuestos Internos, colocó la guinda de la torta, al declarar que esos sobresueldos no eran tributables, lo que significa un ingreso neto adicional para los altos directivos.

\section{Conclusiones}

Mientras que América Latina es un lodazal de corrupción, Chile aparenta ser un islote de probidad. Este panorama es cierto en alguna medida, pero a la vez ocurre que mientras en los otros países, la mayor expresión de corrupción es la coima generalizada, en Chile la coima es excepcional, como lo prueba el reciente Barómetro deValores y Ética Empresarial 2007 aplicada a casi 7000 empleados y gerentes. En esta encuesta el 91\% declara no haber visto nunca repartición de coimas y el 8\% considera que es “a veces” y sólo el 1\% cree que es habitual. En cambio en otros países de la región, la coima es un hábito generalizado y casi público en muchos niveles, mientras que el fraude de fondos fiscales realizado por los burócratas y políticos que ejercen poder, requisito para tomar decisiones que implican el fraude, es la característica esencial de la corrupción en el caso chileno.

Sin embargo, lo preocupante es que la corrupción existe en Chile y asume características mucho más intrincadas y complejas, como las que se describen en esta nota. La superación de esta situación no es la tarea meramente técnica de la Contraloría y otras instituciones, es la difusión de los principios éticos en los partidos políticos y en la participación de la sociedad civil, que por ahora es casi inexistente. Actualmente los medios de comunicación y algunos periodistas son los que han jugado el rol más importante en la lucha en contra de la corrupción. También la Iglesia Católica comienza a interesarse en el tema.

El ministro de Relaciones Exteriores, Alejandro Foxley, ante el llamado de atención de la OECD porque Chile no cumple con las obligaciones para evitar la corrupción, manifestó su total rechazo declarando: "no estoy de acuerdo con eso y estamos sorprendido con lo que dice la OECD", con lo que ratifica la creencia gubernamental de 
que la negación de la realidad hace que ésta sea distinta y desconoce la trascendencia de las leyes de probidad y transparencia que actualmente se discuten en el Parlamento a iniciativa conjunta del gobierno y de la oposición.

\section{Perspectivas para enfrentar la corrupción}

Gobierno y oposición han elegido la estrategia legislativa para detener la corrupción. En un país de exacerbado legalismo como Chile, donde la ley, la buena ley, se vende hasta en los quioscos de periódicos, existe un fetichismo legal que cree que una buena ley resuelve los problemas más complejos. Esta solución se ha aplicado reiteradamente en los últimos 20 años, pro la corrupción va en aumento.

La solución que promueve la oposición es el cambio de gobierno. Según esta concepción, la derecha es la alternativa ya que la corrupción tiene dos fuentes principales: el tamaño del estado y la falta de alternancia. Su ejemplo repetido es México donde el largo gobierno del PRI desarrollo una corrupción masiva. El tercer argumento implícito es que la derecha tiene otros valores, especialmente porque sus líderes que tienen dinero y no necesitan robar.

Estos argumentos son del todo falaces pues hay países con gran alternancia, como era Venezuela, donde el COPEI y Acción Democrática robaban alternadamente, y en el sentido opuesto hay gobiernos de larga duración como los conservadores en el Reino Unido (con Margaret Thatcher) y no hubo corrupción o los gobiernos gaullistas en Francia o los demócratas y republicanos en Estados Unidos. El argumento del tamaño del Estado como causa de la corrupción no calza en absoluto con el caso chileno, pues Chile tiene uno de los estados más pequeños del mundo. Finalmente los valores de la derecha no son muy distintos a los de la Concertación, lo prueba que la máxima corrupción histórica que ha habido en Chile fue durante el gobierno de la derecha con Pinochet. La creencia popular de que los ricos no roban es muy falsa ya que, en gran medida, las grandes fortunas tienen un origen muy turbio, por ejemplo, compras sub valuadas de la empresas públicas, manejo de información confidencial, evasión tributaria (Impuestos Internos señala que hay 12.000 facturas falsa presentadas en las declaraciones impositivas). Lo que ocurre es que roban en grandes volúmenes y lo justifican moralmente porque creen hacer un bien al país porque invierten

Nuestro enfoque es diametralmente opuesto, rechazando también soluciones utópicas como "educar en ética a las nuevas generaciones" o realizar una revolución cultural o la manida solución derechista de la mano dura.

Tampoco es posible contar con el control social porque la dictadura constituyó una sociedad atomizada donde la sociedad civil no tiene nivel orgánico. Tampoco son creíbles las soluciones "técnicas" como crear y fortalecer organismos de control. La Contraloría acaba de digerir un préstamo de varios millones de dólares para "mo- 
dernizarse “ y ya está pidiendo más. El presidente Frei creo un sistema ministerial de auditorías con cientos de técnicos y la corrupción aumentó, gran parte de estos técnicos se especializaron en establecer como presentar rendiciones de cuentas para que no fueran cuestionadas por la Contraloría.

En Chile todos los grandes casos de corrupción han sido denunciados por las asociaciones de funcionarios y trabajadores públicos. Ellos son los agentes estratégicos y su capacitación en ética es esencial para que efectivamente puedan controlar. Sin embargos, normalmente no han recibido ni una hora de capacitación en ética públical' Esta capacitación sólo es válida si es dada a nivel universitario por entidades universitarias estatales, con una definición clara respecto de la ética de los principios y la probidad del cero absoluto.

La experiencia europea y especialmente la norteamericana, muestran que la capacitación en ética es fundamental. En Estados Unidos más de 20.000 funcionarios federales cumplen esta función y ha habido un notable progreso en un país donde la corrupción fue masiva. En definitiva la estrategia factible y exitosa ha sido administrativa.

El otro factor esencial es el rol de la Iglesia, las universidades estatales, las asociaciones de funcionarios y algunos periodistas. Sólo ellos tienen alguna capacidad en la sociedad civil desorganizada. 
Revista de Sociología 21 / 2007 Chile Hoy

Facultad de Ciencias Sociales - Universidad de Chile

\section{Siglas y Acrónimos}

CIADE: Centro de Investigaciones Aplicadas al Desarrollo de la Empresa de la Universidad de Chile.

CORFO: Corporación de Fomento de la Producción.

EFE: Empresa de Ferrocarriles del Estado.

IDECOM: Instituto de Economía de la Universidad de Chile

INDAP: Instituto de Desarrollo Agrícola y Pesquero

MOPT: Ministerio de Obras Públicas y Transportes.

PD Partido Por la Democracia, partido socialdemócrata en el gobierno.

PDC: Partido Demócrata Cristiano, partido socialcristiano en el gobierno.

PS: Partido Socialista, partido socialdemócrata en el gobierno.

RN: Renovación Nacional, partido neoliberal de derecha en la oposición

SENCE; Servicio Nacional de Capacitación y Empleo

\section{Endnotes}

a Patricio Orellana Vargas, Administrador Público, ex profesor de la Universidad de Chile. En su página WEB figuran varios libros y artículos sobre el tema: www. probidadenchile.cl

b Transparencia Internacional Latinoamericana y del Caribe, (TI-LAC), La hora de la Transparencia en América Latina, Berlín, Transparency International, 1996.

c Pasquino, Giancarlo, Corrupción, en Norberto Bobbio, Diccionario de Ciencia Política, México, Siglo XXI Editores, 1988.

d Cálculos nuestros considerando datos de EL MERCURIO y el Instituto Libertad.

e Datos de la Biblioteca del Congreso Nacional.

f El historiador Rafael Luis Gumucio sostiene que la corrupción ha sido parte integrante de la vida republicana y cita los escritos del profesor Venegas (cuyo seudónimo era Valdés Canje). Julio César Jobet también tiene una posición similar.

g Weber, Max, Ensayos de sociología contemporánea, Barcelona, PlanetaAgostini, 1985

h Fliflish, Angel, "En torno a la relación entre moral y política en Max Weber", Santiago, FLACSO, Documento de Trabajo No 17, marzo de 1982.

i Esta norma se le llamó "ley de amarre".

j Weber, opus cit.

$\mathrm{k}$ Esta parte inicialmente era un artículo separado.

1 Universidad Católica, DESUC, "Clima organizacional, Administración Pública”, Santiago, DESUC, 1997. 